\title{
A simple method for slide-mounting chironomid hypopygia in lateral view (and for similar preparations)
}

\author{
Martin Spies \\ SNSB - Zoologische Staatssammlung München, Münchhausenstr. 21, 81247 München, Germany. \\ E-mail:spies@zi.biologie.uni-muenchen.de
}

\section{Introduction}

From the very beginnings of my work in chironomid taxonomy, I have always found it highly informative, frequently even indispensable, to examine and document structures of three-dimensional complexity, such as the adult male hypopygium, from more than just one angle. For example, in the convoluted case of Parachironomus monochromus (van der Wulp) and P. tenuicaudatus (Malloch), sorting specimens and resolving the conflicts among various usages of these names were aided greatly by studying the hypopygia in lateral view (Spies 2000, e.g. figs. 1-4; for further examples, see Spies et al. 1994).

I adopted this method as a student, following what I had seen in collection specimens (Prof. Fittkau and Dr. Reiss used such slides regularly) and in some publications (e.g., Townes 1945, fig. 186A,B; Lehmann 1970). In the literature, however, such models seemed relatively rare, considering how useful I found the approach to be.

One reason for this rarity certainly is that it is much more difficult to keep a hypopygium or abdomen in position on one of its sides until the fluid mounting medium has hardened than it is to lay it down on its wider and flatter venter. A recently discovered easy way to facilitate such preparations is described below.

\section{Material and method}

The chironomid used in the present example is an adult male of an undescribed, novel member of the 'Harnischia complex' of Chironomini genera (listed as Parachironomus sp. 1 „Iijoki“ in Paasivirta 2012); specimen data: FINLAND, Nuuksio National Park, Antiaanpuro (a forest stream); June 2006, Malaise trap, leg. Lauri Paasivirta; kindly donated to ZSM by the collector. Body length prior to slide-mounting was c. 5 $\mathrm{mm}$; the dimensions of the hypopygium (anterior to posterior / left to right / dorsal to ventral) are approx. $400 / 200 / 150 \mu \mathrm{m}$.

Prior to slide-mounting, the specimen's abdomen, or an appropriate rear part of it (in the present case, carefully macerated segments VIII-IX; see Fig. 1), is passed through the series of fluid baths required for the mounting medium to be used. In our example the series led up to concentrated alcohol, and finally to Euparal Essence. Using this commercially available specific solvent and thinning agent avoids the troublesome dispersion of the Euparal drop on the slide that often occurs when a specimen is placed in Euparal directly from alcohol. Whenever working with any substances that evaporate quickly and can be harmful when inhaled - e.g. with concentrated or dilute alcohol or Euparal - be sure to open the containers holding such materials only as briefly as necessary, and keep your workplace sufficiently ventilated.

An entomological minuten pin of a size appropriate for the respective object is selected, and passed through the final stages of the fluid series (in the present case I used a recycled fragment of a size 005 pin that is about $5 \mathrm{~mm}$ long and $0.1 \mathrm{~mm}$ across except at its tapering tip; see Fig. 1). The pin may be inserted in the object at any soaking stage from dilute alcohol to Euparal Essence, whereas inserting it inside a drop of viscous mountant on the slide is messier and incurs greater risk of the object being distorted. If forceps are used during the insertion these must be non-mag-

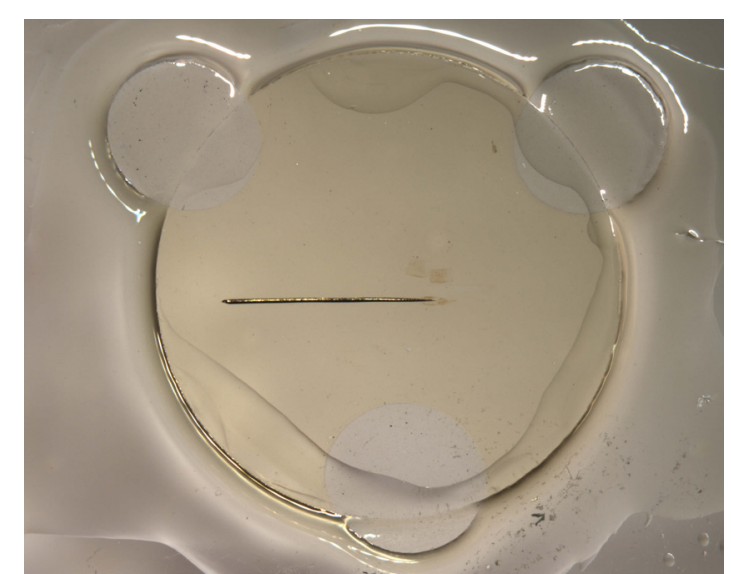

Figure 1. Chironomid adult male abdominal segments VIII-IX mounted in stable lateral view by inserting an entomological minuten pin. Cover slip diameter c. 10 $\mathrm{mm}$; pin length $4.7 \mathrm{~mm}$, max. pin diameter (near left end) c. $100 \mu \mathrm{m}$. Photo: Marion Kotrba \& Martin Spies (ZSM). 
netic, to avoid problems due to the adhesion between pin and forceps.

To minimize rotating or distorting forces being exerted on the object when the cover slip is added, it is advisable to place the latter on supports that are high enough for the object but not too high (see discussion below). In the present example, three commercially available vinyl props of $250 \mu \mathrm{m}$ thickness were used (visible in Fig. 1).

Figure 1 was photographed under a stereoscope (Leica M205 C). Figure 2 is a software composite (using Helicon Focus, version 5.3) of three exposures made under a compound microscope (Zeiss Axioskop 2 plus) in phase-contrast mode. During figure editing (Adobe Photoshop CS4), some specks were removed and background areas smoothened.

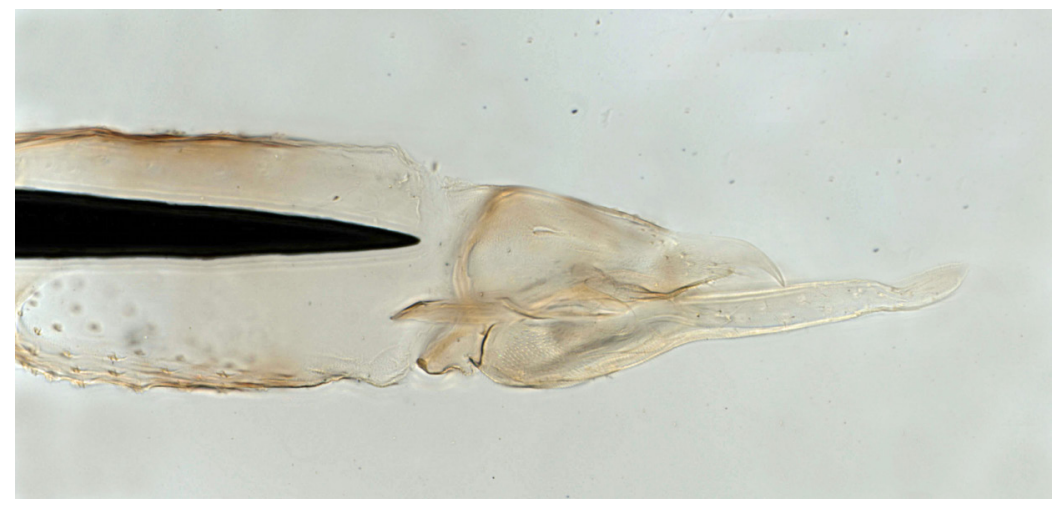

Figure 2. Enlarged view of object in Fig. 1. Image software-integrated from three exposures, then edited for clarity (see text). Photo: Marion Kotrba \& Martin Spies

\section{Discussion} (ZSM).

So far I have used the minuten-anchor method described above for lateral views of hypopygia only, but it is expected to work equally well for mounting other objects that tend to tip over if they are not held in place. Conceivable applications are, e.g., lateral views of dorso-ventrally flattened or curved larval head capsules (possibly best with a few anterior body segments still attached, so that the pin does not enter the head capsule), dorsal views of larval posterior body segments, etc.

Thick mounts, in which the cover slip is relatively far above the glass slide normally used as the base, can limit the selection of microscope objectives through which the object may be examined with sufficient depth of field. A possible remedy is to substitute for the slide by employing another cover slip as the base of the mount (I use commercially available rectangular ones that are $60 \mathrm{~mm}$ long and $24 \mathrm{~mm}$ wide). Embedding an object between two cover slips allows it to be examined at higher magnifications from two sides. For more on this method, including an aluminum 'slide' with central cut-out that reduces risk of breakage during viewing and storage, see Schlee (1966, p. 190 and fig. 2).

\section{References}

Lehmann, J. 1970. Revision der europäischen Arten (Imagines $\lesssim \precsim$ ) der Gattung Parachironomus Lenz (Diptera, Chironomidae). - Hydrobiologia 36(1): 129-158.

Paasivirta, L. 2012. Finnish Chironomidae. - CHIRONOMUS Newsletter on Chironomidae Research 25: 47-70. [http://www.ntnu.no/ojs/index.php/chironomus/issue/view/200]

Schlee, D. 1966. Präparation und Ermittlung von Meßwerten an Chironomidae (Diptera). - Gewässer und Abwässer 41/42: 169-193.

Spies, M. 2000. A contribution to the knowledge of Holarctic Parachironomus Lenz (Diptera: Chironomidae), with two new species and a provisional key to Nearctic adult males. - Tijdschrift voor Entomologie 143(1): 125-143.

Spies, M., Fittkau, E.J. and Reiss, F. 1994. The adult males of Parachironomus Lenz, 1921, from the Neotropical faunal region (Insecta, Diptera, Chironomidae). - Spixiana Supplement 20: 61-98.

Townes, H.K., Jr. 1945. The Nearctic species of Tendipedini (Diptera; Tendipedidae (= Chironomidae)). The American Midland Naturalist 34(1): 1-206. [http://www.jstor.org/stable/2421112] 